\title{
Intracellular product recycling in high succinic acid producing yeast at low $\mathrm{pH}$
}

\author{
S. Aljoscha Wahl ${ }^{1 *} \mathbb{0}$, Cristina Bernal Martinez ${ }^{1,2}$, Zheng Zhao ${ }^{3}$, Walter M. van Gulik and Mickel L. A. Jansen ${ }^{3}$
}

\begin{abstract}
Background: The metabolic engineering of Saccharomyces cerevisiae for the production of succinic acid has progressed dramatically, and a series of high-producing hosts are available. At low cultivation pH and high titers, the product transport can become bidirectional, i.e. the acid is reentering the cell and is again exported or even catabolized. Here, a quantitative approach for the identification of product recycling fluxes is developed.

Results: The metabolic flux distributions at two time-points of the fermentation process were analyzed. ${ }^{13} \mathrm{C}$ labeled succinic acid was added to the extracellular space and intracellular enrichments were measured and subsequently used for the estimation of metabolic fluxes. The labeling was introduced by a labeling switch experiment, leading to an immediate labeling of about $85 \%$ of the acid while keeping the total acid concentration constant. Within $100 \mathrm{~s}$ significant labeling enrichment of the TCA cycle intermediates fumarate, iso-citrate and a-ketoglutarate was observed, while no labeling was detected for malate and citrate. These findings suggest that succinic acid is rapidly exchanged over the cellular membrane and enters the oxidative TCA cycle. Remarkably, in the oxidative direction malate ${ }^{13} \mathrm{C}$ enrichment was not detected, indicating that there is no flux going through this metabolite pool. Using flux modeling and thermodynamic assumptions on compartmentation it was concluded that malate must be predominantly cytosolic while fumarate and iso-citrate were more dominant in the mitochondria.
\end{abstract}

Conclusions: Adding labeled product without changing the extracellular environment allowed to quantify intracellular metabolic fluxes under high producing conditions and identify product degradation cycles. In the specific case of succinic acid production, compartmentation was found to play a major role, i.e. the presence of metabolic activity in two different cellular compartments lead to intracellular product degradation reducing the yield. We also observed that the flux from glucose to succinic acid branches at two points in metabolism: (1) At the level of pyruvate, and (2) at cytosolic malate which was not expected.

Keywords: Metabolic flux analysis, Saccharomyces cerevisiae, ${ }^{13} \mathrm{C}$ labeling, Succinic acid production

\section{Background}

For several products, current microbial hosts can reach very high product titers. Examples are succinic acid, with final product concentrations reaching above $100 \mathrm{~g} / \mathrm{L}$ [1], pyruvate [2], lactic acid [3] and many more. At such high titers and low $\mathrm{pH}$, product transport could become bidirectional, i.e. the product is reentering the cells by back-diffusion or facilitated transport and can potentially be catabolized. Using high succinic acid production as

\footnotetext{
*Correspondence: S.A.wahl@tudelft.nl

1 Department of Biotechnology, Delft University of Technology, Van der Maasweg 9, 2629 HZ Delft, The Netherlands

Full list of author information is available at the end of the article
}

case study, an approach for the identification of product recycling is presented. Succinic acid is an important building block for the production of the biodegradable plastic polybutylene succinate (PBS), polyester polyols, plasticizers and polyurethanes and should replace the petrochemical-derived precursor adipic acid [4]. Commercial scale production has been achieved by several consortia, nevertheless, higher production yields can further strengthen the competitiveness and broaden the application in the market, especially when the oil-price is comparably low.

Metabolic engineering for succinic acid production has focused on the expression of the reductive TCA cycle, glyoxylate cycle, and succinic acid exporters in different 
microbial hosts (see review articles like $[1,5,6])$. Current production strains reach yields of up to $1.74 \mathrm{~mol}(\mathrm{SA}) /$ mol (Glc) [6, 7] and titers above $100 \mathrm{~g} / \mathrm{L}$, which can lead to precipitation at low $\mathrm{pH}$ (solubility limit is $75.5 \mathrm{~g} / \mathrm{L}$ at $25{ }^{\circ} \mathrm{C}$ ). Several groups have focused on E. coli [8-10], an organism which has some drawbacks for the performance in large-scale production vessels [1]. E. coli is sensitive to bacteriophage infections [11] and needs a near-neutral cultivation $\mathrm{pH}$, which requires the addition of stoichiometric amounts of alkali during the fermentation process.

To circumvent the disadvantages of carrying out the fermentation at high $\mathrm{pH}$, companies and research groups have engineered eukaryotic hosts like S. cerevisiae [12], Yarrowia lipolytica [13], or Candida strains [14]. These strains can be cultivated at acidic conditions below the pKa of succinic acid, facilitating the downstream processing (crystallization) and eliminating side products originating from alkali addition, e.g. gypsum [6, 15].

In this study, we analyze the metabolic flux distribution and putative losses due to transport as well as intracellular cycles under production conditions (fed-batch). Especially, labeled product (succinic acid) is used as tracer and modeling approaches are applied to quantify product degradation and recycling. Identification of product recycling and intracellular cycles, including putative parallel routes in different cellular compartments can further improve metabolic engineering strategies and identify relevant product and energy losses.

\section{Methods}

\section{Strain}

The succinic acid producing strain of $S$. cerevisiae used in this study was derived from DSM strain SUC-632, which is described in patent WO2013/004670 [16], and was obtained through classical strain improvement campaigns and the insertion of E. coli fumarase B (fumB, E.C. 4.2.1.2, UniProt accession number P14407). The expression of the fumB gene was controlled by the native TDH1 promoter and the TDH1 terminator. The synthetic expression cassette including appropriate restriction sites was synthesized by GenArt (Regensburg, Germany). This synthetic fragment was cloned in a vector containing a KanMX marker which allows for selection for growth in the presence of G418. The KanMX marker, flanked by lox66 and lox71 sites [37], were removed by the action of Cre-recombinase, as described by Guldender et al. [17]. The fumB cassette and the lox66-KanMX-lox71 sequences were flanked by sequences that allow integration by double cross-over at the YPRCtau3 locus, which is located on chromosome XVI. The fumB and KanMX expression cassettes flanked by YPRCtau3 were isolated from the vector by restriction enzyme digestion and were used for transformations.

Transformants were selected on yeast extract bacto peptone (YEP) 2\% galactose plates supplemented with $200 \mu \mathrm{g} \mathrm{G} 418 / \mathrm{mL}$ for selection of transformants containing the KanMX marker, yielding multiple transformants. Presence of the introduced fumB gene was confirmed by PCR.

\section{Cultivation conditions \\ Seed pre-culture conditions}

The seed-culture was prepared in a shake flask starting from $1.2 \mathrm{~mL}$ of glycerol stocks $(30 \%)$ stored at $-80{ }^{\circ} \mathrm{C}$. The medium composition for the pre-culture was (all in $\mathrm{g} / \mathrm{kg}$ ): carbon source 20 galactose, 2.3 urea, 3.0 $\mathrm{KH}_{2} \mathrm{PO}_{4}, 0.5 \mathrm{MgSO}_{4} \cdot 7 \mathrm{H}_{2} \mathrm{O}$, trace element solution $1 \mathrm{~g} /$ kg (Stock solution: 15.0 EDTA $2 \mathrm{H}_{2} \mathrm{O}, 4.5 \mathrm{ZnSO}_{4} 7 \mathrm{H}_{2} \mathrm{O}$, $1.0 \mathrm{MnCl}_{2} 2 \mathrm{H}_{2} \mathrm{O}, 0.3 \mathrm{CoCl}_{2} 6 \mathrm{H}_{2} \mathrm{O}, 0.3 \mathrm{CuSO}_{4} 5 \mathrm{H}_{2} \mathrm{O}, 0.4$ $\mathrm{Na}_{2} \mathrm{MoO}_{4} 2 \mathrm{H}_{2} \mathrm{O}, 4.5 \mathrm{CaCl}_{2} 2 \mathrm{H}_{2} \mathrm{O}, 3.0 \mathrm{FeSO}_{4} 7 \mathrm{H}_{2} \mathrm{O}, 1.0$ $\mathrm{H}_{3} \mathrm{BO}_{3}, 0.1 \mathrm{KI}$ ) and $1 \mathrm{~g} / \mathrm{kg}$ vitamin solution (Stock: 0.05 Biotin, 1.0 Ca-Pantothenate, 1.0 Nicotinic acid, 25.0 Myo-inositol, 1.0 Thiamine chloride, 1.0 Pyridoxol hydrochloride, $0.2 p$-Aminobenzoic acid). Additionally, $3 \mathrm{~mL}$ of chalk solution $\left(0.1 \mathrm{~g} / \mathrm{g} \mathrm{CaCO}_{3}\right)$ were added to a total of $300 \mathrm{~g}$ of seed pre-culture medium. The pre-culture was finished after 3 days at $30^{\circ} \mathrm{C}$ and $220 \mathrm{rpm}$ reaching a final optical density $(600 \mathrm{~nm})$ of about 7 . All chemicals were obtained from Sigma-Aldrich, with the exception of biotin, pyridoxol hydrochloride, and pantothenate which were obtained from Merck, thiamine from VWR International BV and EDTA was purchased from Roche.

\section{Seed fermentation}

The seed cultivation was performed in a $7 \mathrm{~L}$ bioreactor (Applikon Biotechnology B.V., Delft, The Netherlands) with a maximal working volume of $4 \mathrm{~L}$. The reactor temperature was kept constant at $30{ }^{\circ} \mathrm{C}$; dissolved oxygen tension (DOT) was monitored online using an oxygen probe (Mettler-Toledo, Tiel, The Netherlands). The DO was controlled at a minimum of $20 \%$ air saturation via automatic adjustment of the stirring speed. The bioreactor had an overpressure of $0.3 \mathrm{bar}$, and the aeration rate was $1 \mathrm{vvm}$ (sterile air).

The cultivation was started by transferring $78 \mathrm{~mL}$ of the seed pre-culture (OD 7) into the bioreactor containing $2.4 \mathrm{~L}$ of fermentation medium at $\mathrm{pH} 5$. The medium contained (all in g/kg): 2 glucose, $1\left(\mathrm{NH}_{4}\right)_{2} \mathrm{SO}_{4}, 10 \mathrm{KH}_{2} \mathrm{PO}_{4}$, $5 \mathrm{MgSO}_{4} \cdot 7 \mathrm{H}_{2} \mathrm{O}, 8 \mu \mathrm{L}$ trace element stock solution and $8 \mu \mathrm{L}$ vitamin stock solution (see pre-culture medium). After depletion of the initial carbon source (detected by a decrease in $\mathrm{CO}_{2}$ ), the feed phase (fedbatch) was started. The feed medium contained $500 \mathrm{~g} / \mathrm{L}$ glucose and was 
controlled at an exponential feeding rate of $0.2 \cdot e^{0.1 \cdot t}(\mathrm{~g} /$ $\mathrm{kg} / \mathrm{h}$ ) with a maximum feed rate of $9.2 \mathrm{~g} / \mathrm{kg} / \mathrm{h}$. After about $48 \mathrm{~h}$, a broth weight of $3.69 \mathrm{~kg}$ was reached with a final biomass amount of $53.3 \mathrm{~g}_{\mathrm{CDW}}$.

\section{Production cultivation conditions}

The biomass from the seed cultivation was diluted to OD 15 - this was achieved by transfer of $285 \mathrm{~mL}$ from the seed fermentation, addition of $200 \mathrm{~mL}$ of chalk solution $\left(\mathrm{CaCO}_{3}\right)$ and $2.56 \mathrm{~kg}$ of the production fermentation medium. The medium contained (in $\mathrm{g} / \mathrm{kg}$ ): 1 urea, $1.5 \mathrm{KH}_{2} \mathrm{PO}_{4}, 0.5 \mathrm{MgSO}_{4} \cdot 7 \mathrm{H}_{2} \mathrm{O}, 5$ iron stock solution (in g/kg: $\left.3 \mathrm{FeSO}_{4} \cdot 7 \mathrm{H}_{2} \mathrm{O}, 15 \mathrm{EDTA}\right)$ and 0.001 biotin. This production fermentation phase was running for more than $90 \mathrm{~h}$, at $30{ }^{\circ} \mathrm{C}$, gassed with a mixture of $1: 1$ air: $\mathrm{CO}_{2}$ at a flow of $0.3 \mathrm{vvm}$. During the production phase the DO was controlled at $10 \%$ air saturation. The cultivation feeding rate was initially set to a constant feeding rate of $9.74 \mathrm{~g} / \mathrm{h}$ of a $500 \mathrm{~g} / \mathrm{L}$ glucose solution and was manually decreased at predefined time points ( $>$ to prevent glucose accumulation. To keep the $\mathrm{pH}$ above 3 , chalk solution was added manually at $52 \mathrm{~h}(100 \mathrm{~mL})$, and at $76 \mathrm{~h}$ $(100 \mathrm{~mL})$.

\section{Bioscope labeling experiment}

Aim of the bioscope labeling experiments was to achieve a fast labeling gradient in the extracellular space without disturbing the metabolic steady-state. The experiments were performed during two phases of the production phase. The BioScope [18], a plug-flow 'satellite' reactor, was connected to the fed-batch production cultivation (see Fig. 1). The bioscope reactor inflows were set at the following rates: (1) feeding solution at $0.56 \mathrm{~mL} / \mathrm{min},(2)$ inflow from the bioreactor: $0.14 \mathrm{~mL} / \mathrm{min}$.

These rates lead to a 5 -fold dilution of the bioreactor broth, including the biomass concentration. The concentrations of glucose and succinic acid in the BioScope feed were chosen the same as the extracellular concentrations, to avoid changes in extracellular concentrations after the dilution. Equivalent to the bioreactor a mixture of 1:1 $\mathrm{CO}_{2}$ and air was passed through the gas channel of the BioScope. To mimic the flow of $0.5 \mathrm{vvm}$ in the bioreactor a flow rate of $0.086 \mathrm{~L} / \mathrm{min}$ was used.

\section{Intracellular and extracellular sampling}

Samples for intracellular metabolite quantification were withdrawn using a dedicated rapid sampling device (for bioreactor samples) and quenched in $6 \mathrm{~mL}-40{ }^{\circ} \mathrm{C}$ pure methanol [19]. From this sample $1 / 3$ was further processed-the quenched biomass was poured on a precooled filter and the filter cake was washed with $30 \mathrm{~mL}$ methanol cooled at $-40{ }^{\circ} \mathrm{C}$ [20]. The filter was rapidly transferred into a falcon tube containing $15 \mathrm{~mL}$ of $75^{\circ} \mathrm{C}$ ethanol and $120 \mu \mathrm{L}$ of ${ }^{13} \mathrm{C}$ cell extract was added. Extraction of the intracellular metabolites was achieved by water bath boiling $\left(3 \mathrm{~min}\right.$ at $\left.95^{\circ} \mathrm{C}\right)[21]$.

Samples from the BioScope were collected for about $2 \min (\sim 1.4 \mathrm{~mL})$ at each port, weighted and the complete volume was processed by filtration and extraction as in the case of a bioreactor sample.

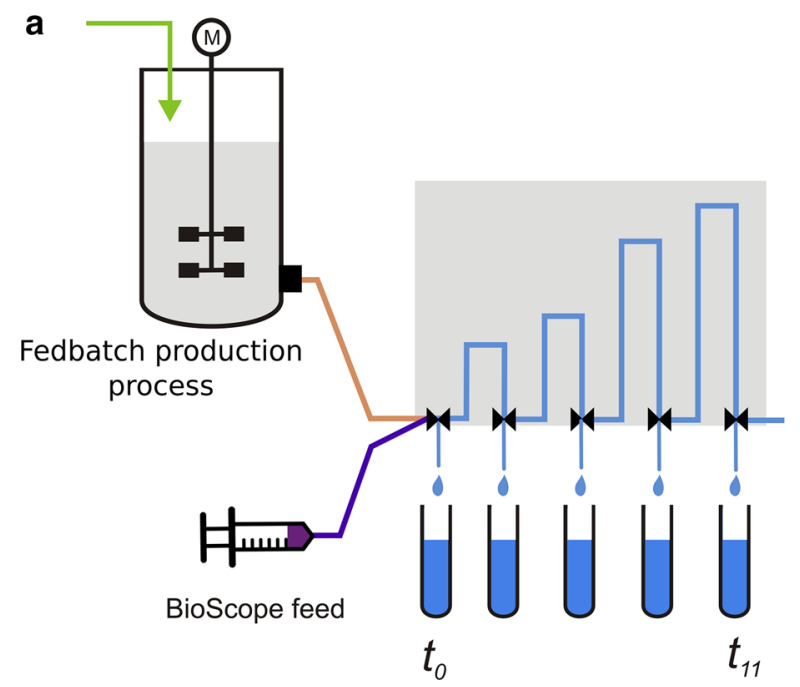

b
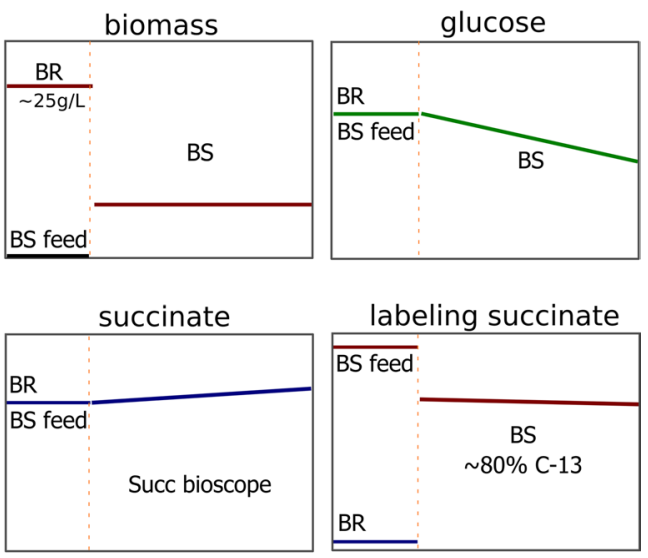

Fig. 1 Setup of the bioscope labeling experiment. a The bioscope has inflows from the bioreactor (flow $0.14 \mathrm{~mL} / \mathrm{min}$ ) and the BioScope feed $(0.56 \mathrm{~mL} / \mathrm{min})$. b The BioScope feed contains labeled succinic acid matching the concentration of succinic acid of the bioreactor broth. The solution also matches the residual glucose concentration (unlabeled). This setup leads to a dilution of the biomass concentration while the residual concentration of glucose and succinic acid are similar to the BR cultivation. Using labeled succinic acid allows for immediate labeling with about $80 \%$ enrichment 
Extracellular samples from the BioScope were obtained by vacuum filtration [18]. A vial connected to a vacuum pump was equipped with a syringe filter and placed at the bioscope port for about $1.5 \mathrm{~min}$ to collect about $1.0 \mathrm{~mL}$ of filtrate. The sample was stored at $-80{ }^{\circ} \mathrm{C}$ until further processing. To determine the concentration in the extracellular space, $100 \mu \mathrm{L}$ of the filtrate was transferred to a GC vial containing $20 \mu \mathrm{L}$ of ${ }^{13} \mathrm{C}$ cell extract as internal standard and processed comparable to intracellular samples.

\section{Model setup}

A model was developed based on the stoichiometry and atom-transitions listed in Additional file 1: Table S1. The model equations were generated automatically based on model definition files. The labeling of extracellular succinic acid was introduced as initial value for the respective labeling state variables for succinic acid.

Compartmentation was taken into account by the introduction of a cytosolic and a mitochondrial metabolite pool (index_cyt and_mit). The simulation will predict separate enrichment patterns. The concentration of each pool depends on its distribution $f$. The variables f_cyt_Succ, f_cyt_Mal, etc. reflect the (amount) fraction present in the cytosol. All intracellular rates are described as rate per amount of biomass dry weight $\left(\mathrm{g}_{\mathrm{CDW}}\right)$ and pools are balanced as amounts per biomass $(\mu \mathrm{mol} / \mathrm{g})$.

The network consists of 31 balanced metabolites, and 39 net- and 24 exchange fluxes (the complete model is documented in Additional file 1, B). Additionally, there are seven parameters for the distribution of TCA cycle intermediates between cytosol and mitochondria. Four fluxes were measured (glucose uptake, and succinate, malate and glycerol production), resulting in 28 fluxes to be estimated. Several (8) of those were excluded from the parameter estimation and set to a fixed value, as these could not be determined from the observed enrichment patterns (and in turn had no impact on the enrichment pattern). Especially, glycolytic exchange fluxes or exchange fluxes in the pentose phosphate pathway were not identifiable as no labeling enrichment was observed within the given observation time. The complete network and a priori fixed fluxes can be found in Additional file 1.

\section{Simulation and parameter estimation}

All simulations and parameter estimations were performed using the simulation software gPROMS 4.0 [22]. The gPROMS model was generated based on the network stoichiometry and known atom transitions using a selfdeveloped model parser.

The parameter estimation was performed using the implemented NLSQ solver. The flux estimation was manually repeated from different starting values and typically converged to comparable minima solutions, especially for fluxes that were estimated with high confidence (see Additional file 1: Tables S2, S3). The confidence intervals were calculated based on linear error propagation (i.e. linearization of the equation system at the measurement timepoints) that is integrated in the gPROMS software package. For the mass isotopomer measurements an error of 3\% (absolute) was assumed. Exception is the measurement of Oaa which was less reliable because of the very low concentration of Oaa. Here 30\% error were used. This estimated errors not only reflects the MS noise but also the non-ideal plug-flow within the BioScope reactor [23], i.e. the sampling time point is a (narrow) distribution of different time points. The errors of the MS/MS measurements itself are estimated to be between 0.5 and $2 \%$ [24]. The standard deviation of the biomass specific extracellular rates was also calculated using linearized error propagation of the black-box model using PWA rate functions. The required standard deviations of the observables (i.e. succininc acid, biomass and other concentrations) was calculated from the repetitive measurements, i.e. the average standard deviation of all observed timepoints.

\section{Results}

\section{Extracellular metabolic rates}

The rates of substrate uptake, succinic acid production and by-product formation were determined from the medium inflow and concentration measurements in time of the bioreactor cultivation using a dynamic flux estimation approach (see Table 2; Additional file 1, A). During the initial phase of the production process $(0-24 \mathrm{~h})$, there is biomass growth at an average rate of $0.07 \mathrm{~h}^{-1}$ (Fig. 2). During this phase, the glucose uptake rate is high (average of about $1.5 \mathrm{mmol} / \mathrm{g} / \mathrm{h}$ ) and then decreases till the end of the cultivation. Similarly, the succinic acid production rate decreases in time while malic acid is excreted at a constant rate during the process. These rates reproduce the measured concentration profile (Additional file 1: Figure S1). Please note that the residual glucose concentration is the result of feed and uptake rates and reacts very sensitive due to the high biomass concentration and rates. Thus, the deviations observed in Additional file 1: Figure S1 actually reflect small (relative) misfits of the uptake rate.

\section{Intracellular metabolite concentrations}

The intracellular metabolite quantifications show that the levels of three intermediates of the TCA cycle are significantly higher than the others, namely succinate, malate and citrate (Table 4). Compared to wild-type strains at similar glucose uptake rate [25], the concentrations are 

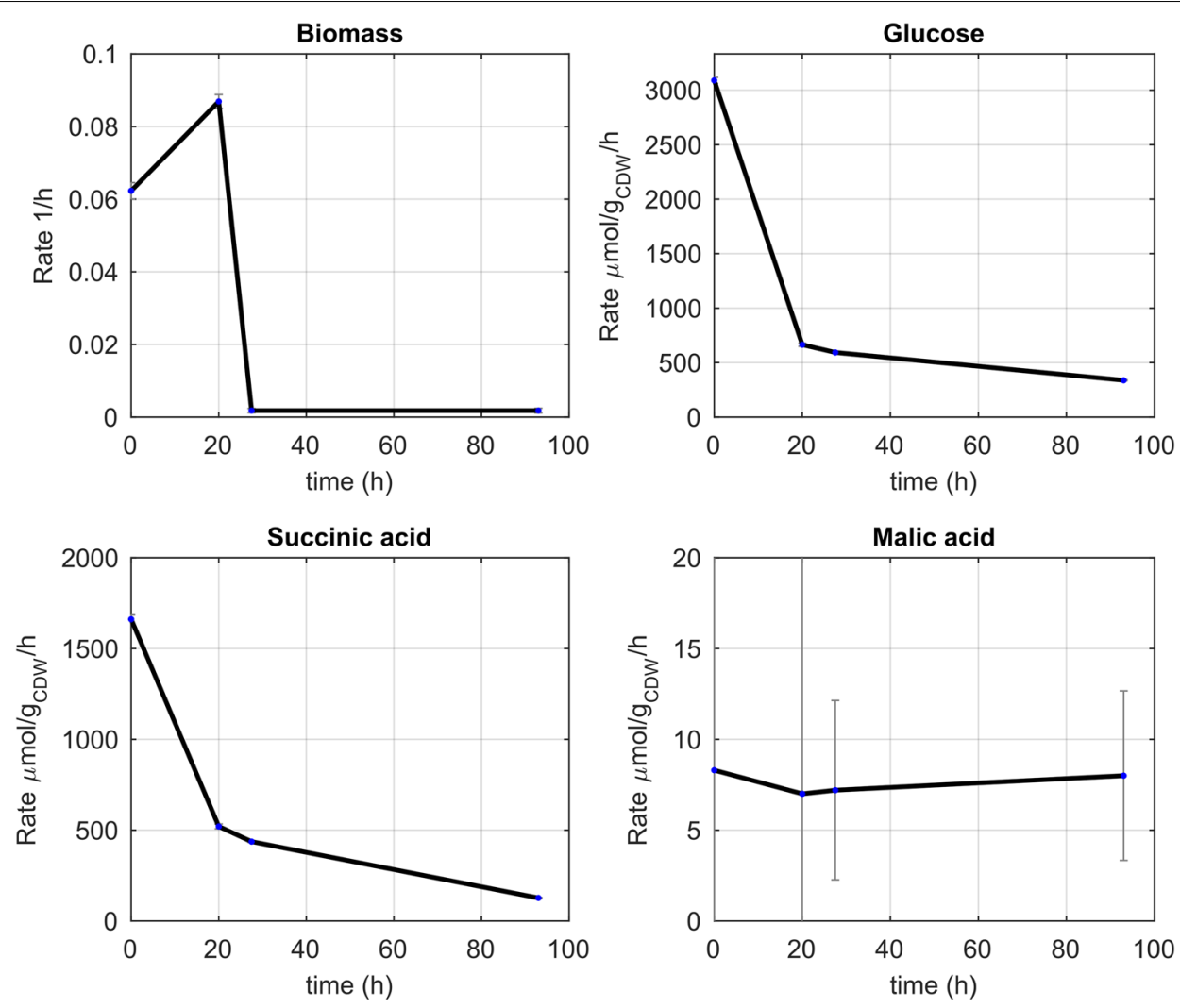

Fig. 2 Estimated biomass specific rates over the cultivation time. Table 2 displays the rates at the time of the bioscope experiments. Standard deviations are calculated using linear error propagation (i.e. linearization of the ODE model)

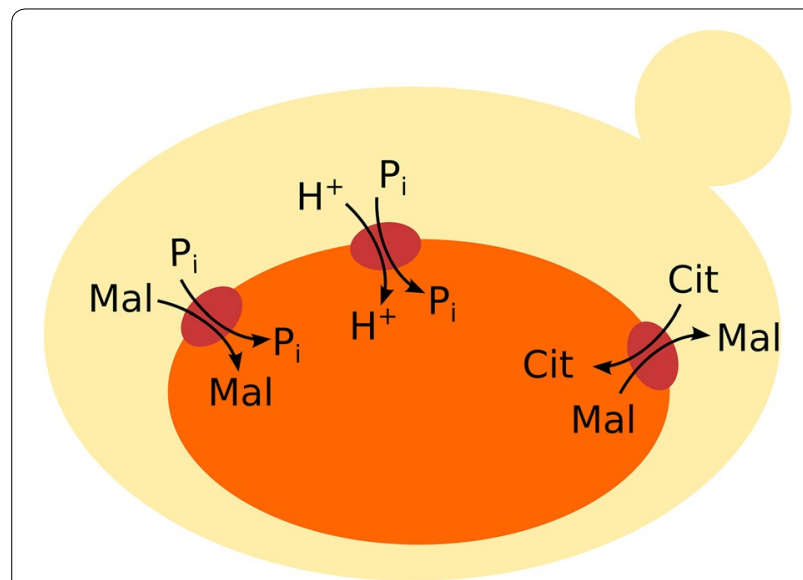

Fig. 3 Scheme of intracellular transport reactions of TCA cycle intermediates. A complete list of assumed transport reactions can be found in Table 1, estimated rates are displayed in Figs. 5 and 7

100, 50 and 4-fold increased for succinate, malate and citrate, respectively. The concentration of these metabolites seems to increase with increasing extracellular succinate. Conversely intracellular pyruvate as well as $\alpha$-ketoglutarate decrease with production time and product concentration.

\section{Identification of product recycling fluxes}

Earlier findings suggested that there is a high bidirectional flux of succinic acid over the cellular membrane [26]. This observation was exploited in this study by introducing the ${ }^{13} \mathrm{C}$ tracer via succinic acid, labeled on $\mathrm{C}_{1}$ and $\mathrm{C}_{4}$. Wash-in labeling experiments were performed at two time points of the cultivation, 52 and $73 \mathrm{~h}$, e.g. during the mid-production and late production phase respectively. The intracellular and extracellular metabolite concentrations in the bioreactor were measured before and after the bioscope experiment which took about $1.5 \mathrm{~h}$. At the mid production stage $(\mathrm{t}=52 \mathrm{~h})$ an extracellular succinic acid concentration of $490 \mathrm{mmol} / \mathrm{L}$ was reached, and $590 \mathrm{mmol} / \mathrm{L}$ at $\mathrm{t}=73 \mathrm{~h}$. These concentrations were used for the bioscope feed medium. For the estimation of intracellular fluxes, (quasi) steady-state was assumed during the labeling experiment. Please note that the concentration as well as the production flux of succinic acid changed less than $2 \%$ during the labeling experiment $(1.5 \mathrm{~h})$. 


\section{Compartmentation of the TCA cycle intermediates}

The distribution of intracellular TCA cycle intermediates between the cytosol and mitochondria cannot be determined by direct measurements. Citrate (Cit), isocitrate (iCit), $\alpha$-ketoglutarate (aKG), succinate (Succ), malate (Mal), fumarate (Fum) and oxaloacetate (Oaa) are present in the mitochondria as well as in the cytosol. The distribution can significantly influence the metabolic engineering strategy [27]. Transport studies suggest that there are several transport proteins involved in shuttling metabolites between the cytosol and mitochondria, especially the phosphate/proton symport, Mal/Pi carrier, aKG/Mal carrier and Cit/Mal carrier (Fig. 3, Table 1 and references therein).

Assuming that these transporters operate close to equilibrium, the distribution of all TCA cycle intermediates can be calculated (see Additional file 1 for detailed calculations and assumptions). Please note that these calculations are subject to assumptions, i.e. transport equilibrium, subcellular volume distribution and electrochemical potentials. The calculations are used to obtain a theoretical reference for comparison, but were not used in the flux estimation.

\section{Mid-production phase $(\mathrm{t}=\mathbf{5 2} \mathrm{h})$}

The succinic acid labeling nearly reaches the extracellular enrichment at the first sampling time point $(t=18 \mathrm{~s})$, this indicates a very rapid exchange between intra- and extracellular succinic acid pools. A fast increase in ${ }^{13} \mathrm{C}$ enrichment was also observed for fumarate, reaching $28 \%(+2)$ after about $180 \mathrm{~s}$ (Fig. 4). Additionally, enrichment is observed in isocitrate and $\alpha$-ketoglutarate, reaching 11 and $18 \%$, respectively. Note that for $\alpha$-ketoglutarate the labeling is observed for the +1 trace, while it is +2 for isocitrate which is in agreement with the decarboxylation. The enrichment in +1 $\alpha$-ketoglutarate and +2 isocitrate from succinate as labeling source can only be obtained via the oxidative TCA cycle, or a reverse isocitrate lyase, and malate synthase for providing glyoxylate (or alternative enzymes
[28]). In both cases, malate is required, with the difference that labeled malate would only be required for the full oxidative cycle. From the experimental measurements, malate reaches a maximal enrichment of about $2 \%(+2)$, citrate about $5 \%(+2)$. This discrepancy in labelling enrichments of intermediates suggest the compartmentalization of oxidative and reductive TCA cycle activities.

Furthermore, fumarase is a reversible reaction with an equilibrium constant $\left(K_{e q}\right)$ of about 4 (for the oxidative direction). To obtain malate from fumarate, the ratio $\mathrm{Mal} / \mathrm{Fum}$ has to be below 4 . The intracellular measurements show values between 150 and 185 , for $\mathrm{t}=52 \mathrm{~h}$ and $\mathrm{t}=73 \mathrm{~h}$ respectively (Table 2 ). In an extreme scenario to enable oxidative and reductive directions, all fumarate would be in the mitochondria and (nearly) all malate in the cytosol (scenario 2 in Table 3).

In the cytosol malate is synthesized from glucose, which will have a natural enrichment $\left(1.1 \%{ }^{13} \mathrm{C}\right)$. In contrast to cytosolic malate, mitochondrial malate will mostly originate from the oxidative TCA cycle, carrying labeling from succinate, but because of the small amount compared to the cytosolic amount, no labeling is observed in the whole cell measurements. In the case of isocitrate, fumarate and $\alpha$-ketoglutarate there seems to be an accumulation in the mitochondria and therefore ${ }^{13} \mathrm{C}$ enrichment can be observed from the whole-cell measurements.

The enrichment in oxaloacetate has been measured as well, however, because of the low concentration in the cell as well as instability during the extraction, the measurements contain significant noise. Nevertheless, a fast enrichment trend can be observed that is further supported by the fast enrichment seen in the associated amino acid aspartate. The labeling enrichment in aspartate (resp. oxaloacetate) also renders a putative role of a reverse glyoxylate shunt, because labeling in oxaloacetate could only be obtained from malate. Summarizing, the labeling observations can only be reproduced by a metabolic network that especially features:

Table 1 Implemented mitochondrial transporters

\begin{tabular}{llll}
\hline Gene & Description & Reaction & Reference \\
\hline YMR241 W & Citrate and oxoglutarate carrier protein & akg_cyt + cit_mit $\rightarrow$ akg_mit + cit_cyt \\
YBR291C & Citrate transport protein & cit_cyt + mal_mit $\Leftrightarrow$ cit_mit + mal_cyt \\
YBR291C & Citrate transport protein & cit_cyt + icit_mit $\Leftrightarrow$ cit_mit + icit_cyt \\
YLR348C & Mitochondrial dicarboxylate carrier & mal_cyt + pi_mit $\Leftrightarrow$ mal_mit + pi_cyt \\
YKL120 W & Oxaloacetate carrier & h_cyt + oaa_cyt $\Leftrightarrow$ h_mit + oaa_mit \\
YLR348C & Mitochondrial dicarboxylate carrier & pi_mit + succ_cyt $\rightarrow$ pi_cyt + succ_mit \\
YJR095 W & Mitochondrial succinate-fumarate transporter & fum_mit + succ_cyt $\rightarrow$ fum_cyt + succ_mit
\end{tabular}

The reactions were derived from the genome scale model iMM904 [33] and references found for the respective genes 

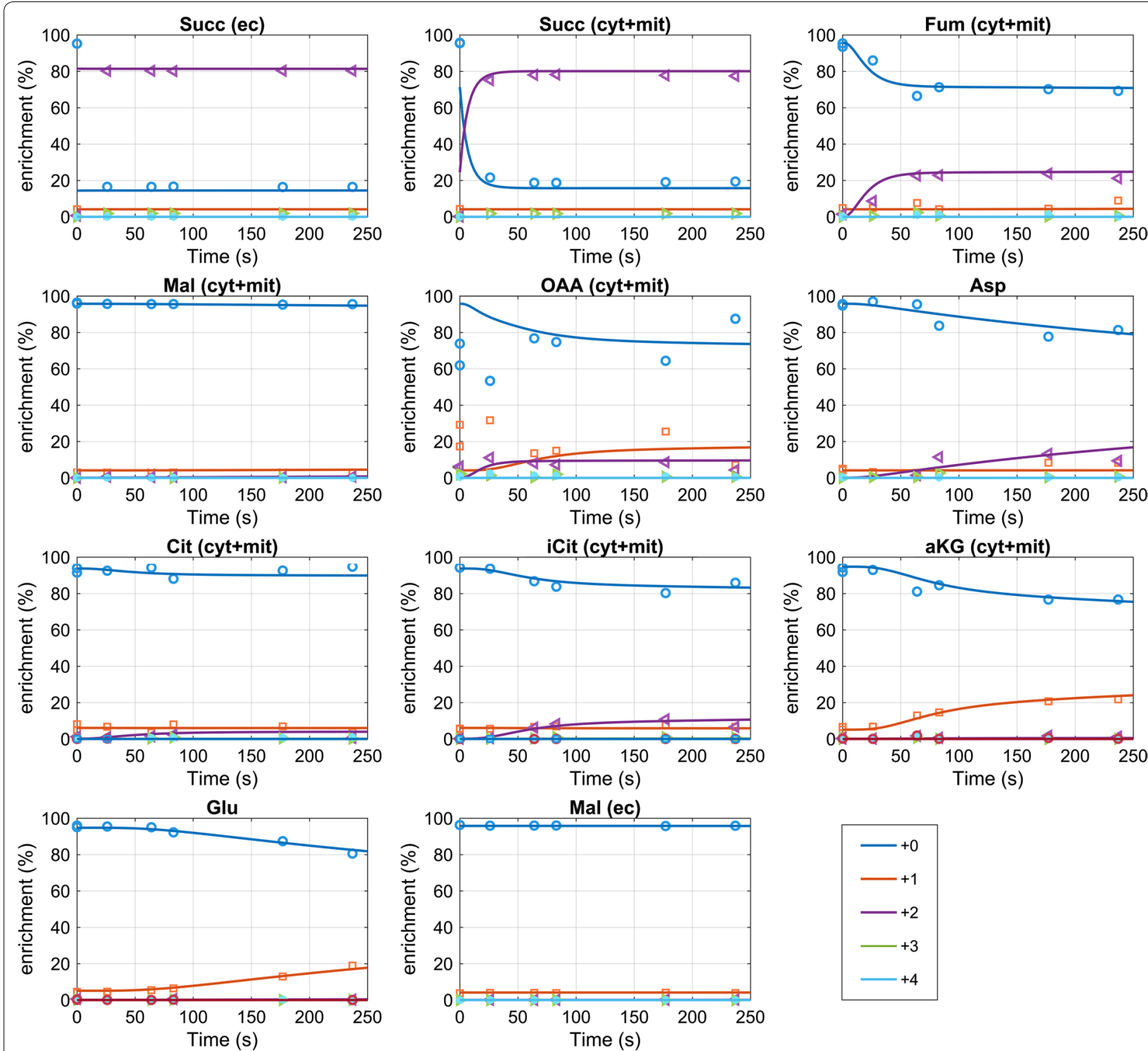

Fig. 4 Enrichment measurements and mass isotopomer simulation for $t=52 \mathrm{~h}$. The mass isotopomer simulation represents the sum of the mitochondrial and cytosolic prediction. The measurements for Oaa were less accurate because of the low intracellular concentration and were included in the parameter estimation with a 10x higher standard deviation. The mass isotopomer measurements can be found in Additional file 1: Table S6

Table 2 Biomass specific conversion rates at the time points of the BioScope experiments

\begin{tabular}{lllll}
\hline $\begin{array}{l}\text { Growth } \\
\mathbf{1 / h}\end{array}$ & $\begin{array}{l}\text { Glucose } \\
\boldsymbol{\mu m o l} / \mathbf{g} / \mathbf{h}\end{array}$ & $\begin{array}{l}\text { Succinic acid } \\
\boldsymbol{\mu m o l} / \mathbf{g} / \mathbf{h}\end{array}$ & $\begin{array}{l}\text { Malic acid } \\
\boldsymbol{\mu m o l} / \mathbf{g} / \mathbf{h}\end{array}$ & $\begin{array}{l}\text { Glycerol } \\
\boldsymbol{\mu m o l} / \mathbf{g} / \mathbf{h}\end{array}$ \\
\hline $\mathrm{t}=52 \mathrm{~h}$ & & & & \\
$\quad 0.0018 \pm 0.0006$ & $497.4 \pm 5.3$ & $320.7 \pm 3.5$ & $7.5 \pm 4.8$ & $0.9 \pm 0.2$ \\
$\mathrm{t}=73 \mathrm{~h}$ & & & & \\
$0.0018 \pm 0.0006$ & $415.6 \pm 4.7$ & $221.3 \pm 3.0$ & $7.8 \pm 4.7$ & $1.0 \pm 0.2$
\end{tabular}

The standard deviations are calculated from linear error propagation using the blackbox model with piecewise linear rate functions
- A fast exchange of succinic acid over the plasmaas well as mitochondrial membrane (here about $15 \mathrm{mmol} / \mathrm{g}_{\mathrm{CDW}} / \mathrm{h}$ ),

- a differential distribution of TCA cycle intermediates between the cytosol and mitochondria,

- a fast exchange of the intermediate oxaloacetate with aspartate, as well as $\alpha$-ketoglutarate with glutamate.

Specifically, the parameter estimation suggests that malate, succinate and citrate are predominantly ( $\geq 95 \%)$ cytosolic (in amount). Oxaloacetate, $\alpha$-ketoglutarate, 
fumarate and isocitrate are in the range of $40-65 \%$ (Table 5).

At this stage of the cultivation growth is basically absent (due to nitrogen depletion) and the substrate glucose is used for (by-)product formation and maintenance only. At the pyruvate branch point about $1 / 3$ of the carbon enters the reductive route (Fig. 5). The oxidative (mitochondria) and reductive (cytosolic) routes interchange at the level of malate, fumarate as well as succinate. The flux estimation suggests that about $11 \%$ of the produced cytosolic malate enters the mitochondria and returns in the form of succinate resp. is catabolized to $\mathrm{CO}_{2}$ (this cannot be discriminated). On the one hand, this can be attributed to stoichiometric coupling, i.e. succinate taken from the TCA cycle has to be replenished. On the other hand, the exchange of malate seems reasonable in view of the high intracellular (cytosolic) concentration (Tables 4, 5). The import of malate into the mitochondria leads to a lower flux of malate to fumarate in the cytosol. This finding together with the surprisingly high malate concentration, as well as observing malate as by-product suggest a putative limitation of fumarase in the reductive direction. Although, there is a high thermodynamic driving force, not all malate arriving from oxaloacetate can be converted to fumarate, but is diverted to the TCA cycle and also the extracellular space.

From the model estimation, the labeling originating from the extracellular labeled succinic acid leads to a high labeling in cytosolic succinate, that interchanges with the mitochondrial pool using the Suc/Pi carrier but also Suc/ Fum transporter. The predicted labeling of mitochondrial succinate is $50 \%$. For the downstream metabolites Fum, Mal and Oaa lower enrichments are predicted because of the inflow of unlabeled metabolites from the cytosol, especially the exchange with Fum and Mal.

Unfortunately, but also expected, the parameter estimation has large confidence intervals (Additional file 1: Table S2) and there is significant correlation between

Table 4 Intracellular metabolite amounts $\left(\mu \mathrm{mol} / \mathrm{g}_{\mathrm{CDw}}\right)$

\begin{tabular}{lllllllll}
\hline Time $(\mathbf{h})$ & Succ & Fum & Mal & Cit & iCit & aKG & Pyr & Glx \\
\hline 51.3 & 71.8 & 0.42 & 63.8 & 25.0 & 0.80 & 1.41 & 0.45 & 0.18 \\
72.6 & 94.6 & 0.50 & 92.7 & 35.4 & 1.76 & 0.93 & 0.24 & 0.13 \\
\hline
\end{tabular}

The measurements were performed just before the bioscope labeling experiment (single measurement at port 0)

Table 3 Thermodynamic comparison of different scenarios (1) Equilibrium of Mal/Pi transport (2) Derived from the assumption of oxidative fumarase in the mitochondria (Mal/Fum $=3.5$ )

\begin{tabular}{|c|c|c|c|c|}
\hline & \multicolumn{2}{|c|}{ Mal/Pi at equilibrium } & \multicolumn{2}{|c|}{ Mitochondrial fumarase in oxidative direction } \\
\hline & Conc. ratio $(M / C)$ & Cytosolic amount (\%) & Conc. ratio $(\mathrm{M} / \mathrm{C})$ & Cytosolic amount \\
\hline Malate & 9.83 & 50 & 0.236 & $98.1 \%$ \\
\hline Succinate & 9.59 & 51 & & \\
\hline Fumarate & 9.97 & 50 & Inf & 0 \\
\hline Citrate & 25.51 & 28.1 & 0.612 & $93.9 \%$ \\
\hline Isocitrate & 25.41 & 28.2 & 0.610 & $93.9 \%$ \\
\hline aKG & 9.96 & 50.1 & 0.239 & $97.6 \%$ \\
\hline
\end{tabular}

The equilibrium calculations depend on assumptions on the electrochemical potential $(\mathrm{pmf}=-180 \mathrm{mV})$, the $\mathrm{pH}$ difference $(\Delta \mathrm{pH}=0.5)$ and volume distribution (cytosol 70\%, mitochondria 7\%)

Table 5 Estimated distribution of TCA cycle acids during the fed-batch process $(t=52 h$ and $t=73 h)$

\begin{tabular}{|c|c|c|c|c|}
\hline & $\%$ Amount cytosol 52 h (\%) & $\%$ Amount cytosol 73 h (\%) & Conc. ratio $\mathrm{M} / \mathrm{C} 52 \mathrm{~h}$ & Conc. ratio $\mathrm{M} / \mathrm{C} 73 \mathrm{~h}$ \\
\hline aKG & 40.0 & 40.0 & 15.0 & 15.0 \\
\hline Cit & 94.9 & 96.6 & 0.5 & 0.3 \\
\hline FUM & 40.0 & 95.5 & 15.0 & 0.5 \\
\hline iCit & 62.5 & 78.2 & 6.0 & 2.8 \\
\hline Mal & 99.6 & 99.8 & 0.0 & 0.0 \\
\hline Oaa & 54.7 & 89.9 & 8.3 & 1.1 \\
\hline Suc & 99.8 & 99.8 & 0.0 & 0.0 \\
\hline
\end{tabular}

Please note, the concentration ratio depends on assumptions for the subcellular volumes (here cytosol 70\%, mitochondria $7 \%$ ) 


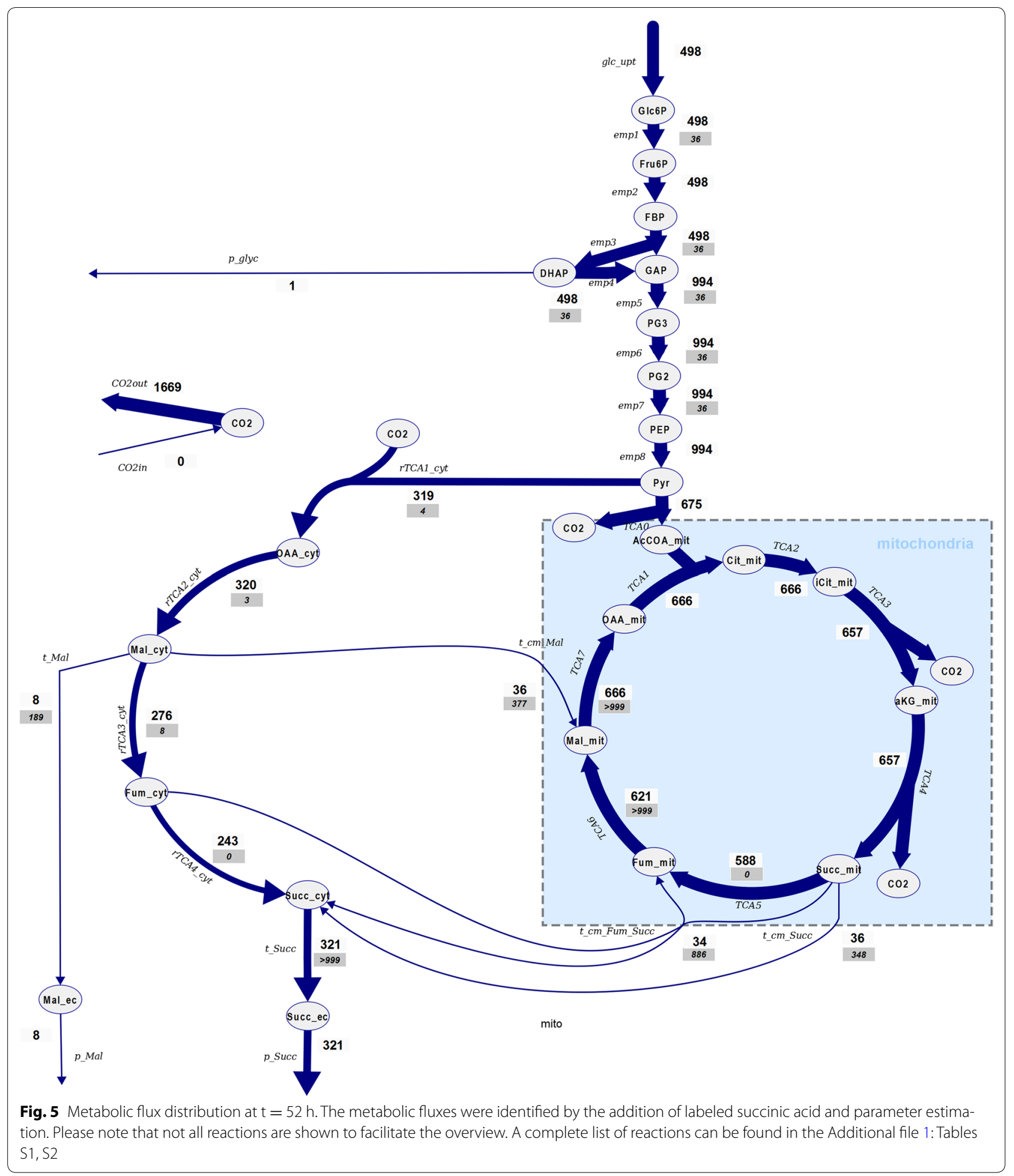

transport (exchange) rates and the metabolite distribution variables. To further analyze if the results are consistent, a thermodynamic analysis was performed using the estimated metabolite distribution, compartment volume distribution and previously reported estimates of cofactor ratios (Additional file 1: Table S4). It can be seen that for most reactions the flux direction estimated from the ${ }^{13} \mathrm{C}$ model is supported, nevertheless, there is 

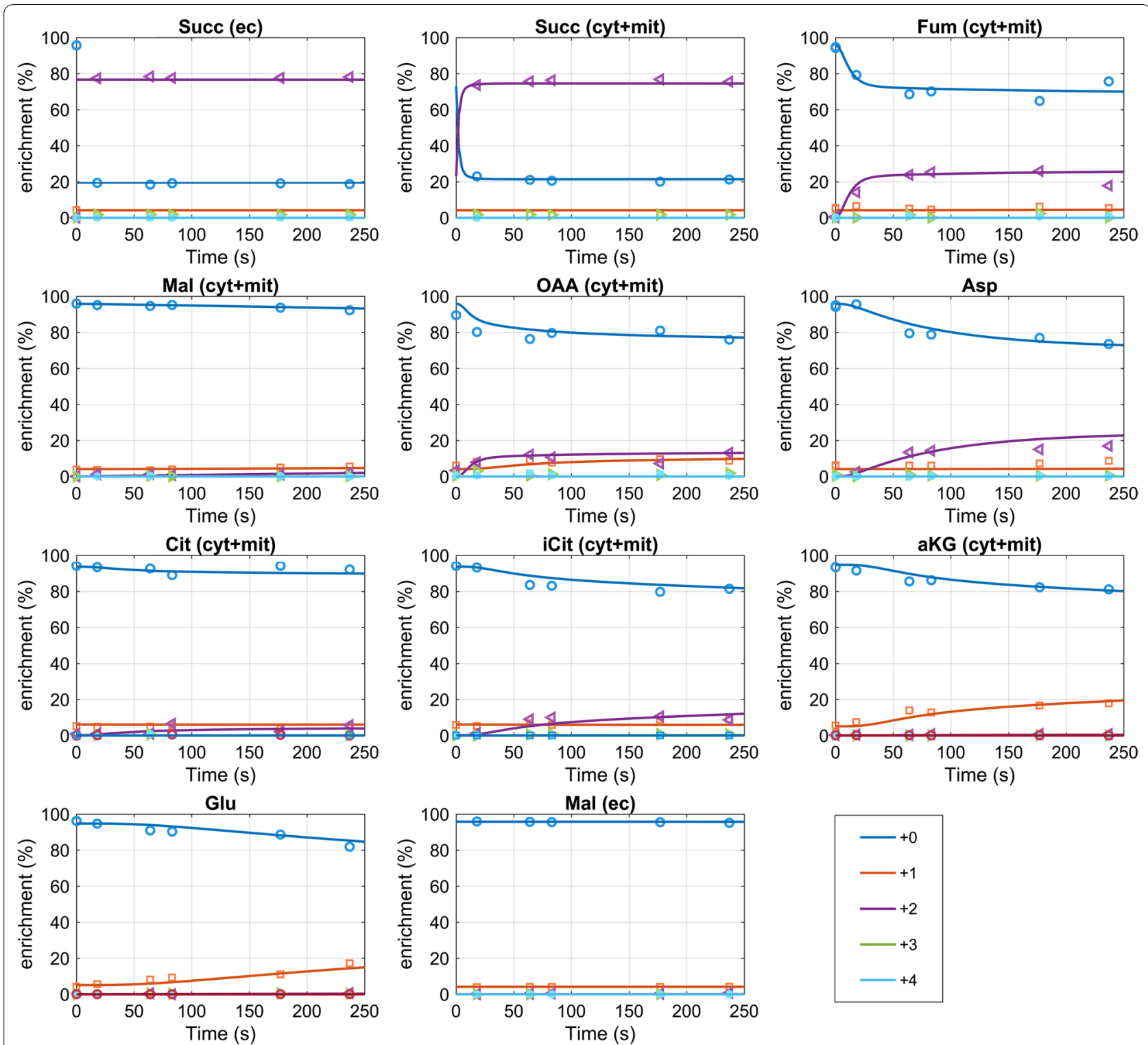

Fig. 6 Simulated and measured mass isotopomers for $t=73 \mathrm{~h}$. From the simulation the sum of mass isotopomers from mitochondria and cytosol are calculated, representing the predicted whole-cell measurements. The mass isotopomer measurements can be found in Additional file 1: Table S7

an inconsistency for mitochondrial succinate dehydrogenase (the estimated Fum/Suc ratio is too high). The transport reaction directions are consistent with the flux estimation, except the direction of the Fum/Suc shuttle-for Suc and Fum the largest standard deviations were observed.

Despite the uncertainty and the observed inconsistencies, it has to be noted that the observed labeling requires mechanisms to enable oxidative and reductive TCA cycle activity together with exchange reactions. The available measurements currently only allow an estimation with inaccuracies.
Late production phase $(t=73 \mathrm{~h})$

In the late production phase the glucose consumption rate is reduced and the rate and yield of succinic acid decreased. Compared to the mid-production phase, a lower fraction of pyruvate is entering the reductive, cytosolic product pathway. About $68 \%$ of the available pyruvate enters the mitochondria and is catabolized in the oxidative TCA cycle. There is some exchange of succinate and fumarate between the cytosol and the mitochondria, i.e. some cytosolic Fum is entering the mitochondria and succinate is excreted to the cytosol, maybe because of a limitation in mitochondrial fumarate reductase flux $(Q /$ 


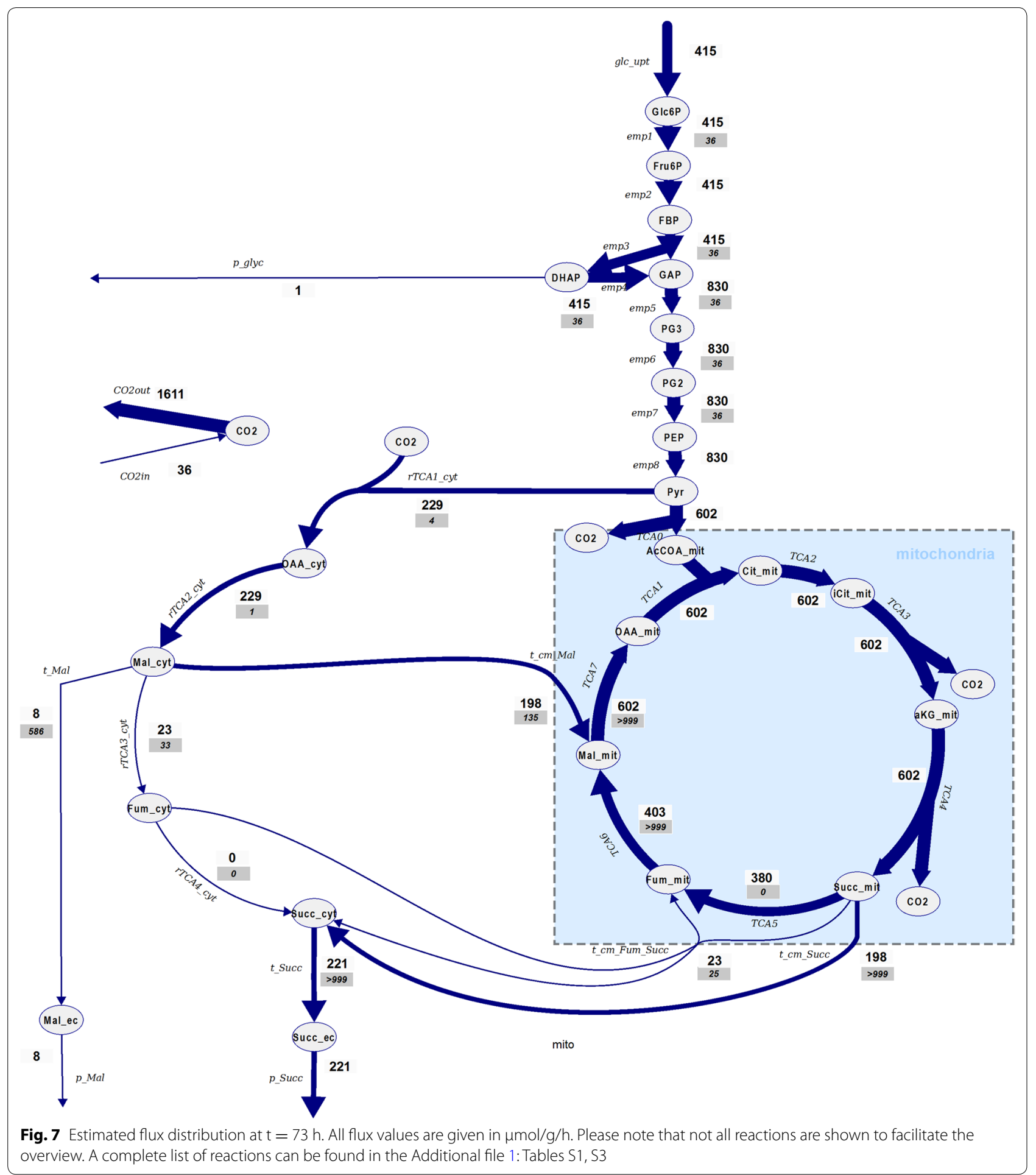

$K_{e q}>1$, Additional file 1: Table S5). Additionally, there is a high bidirectional rate for the Suc/Pi carrier, but low net flux. This constellation leads to a lower cytosolic activity of fumarate reductase compared to the previous reaction steps, i.e. fumarase.
Comparing the estimated distribution of acids to the previous phase, the general trend is comparable, but the cytosolic amount of Fum and Oaa is higher (Table 5). There is no observable enrichment in malate, and also citrate only reached a lower enrichment of $2 \%$ for the +2 
mass. In contrast, $\alpha$-ketoglutarate +1 reaches $21 \%$ after $237 \mathrm{~s}$ (Fig. 6) indicating a pool mostly participating in the oxidative TCA cycle (estimated 15 times higher concentration in the mitochondria compared to the cytosol). Isocitrate is reaching $10 \%$ enrichment on the +2 mass trace, similar to the mid-production phase.

The flux estimation shows a significant change in metabolic flux distribution, i.e. $86 \%$ of the synthesized cytosolic malate now enters the mitochondria. During this phase, the export of malate to the extracellular space is at a comparable rate $\left(8 \mu \mathrm{mol} / \mathrm{g}_{C D W} / \mathrm{h}\right)$, in relative amounts about $3.4 \%$ which is a slight increase compared to the earlier phase (Fig. 7). The intracellular malate concentration at this stage is about $92 \mu \mathrm{mol} / \mathrm{g}_{\mathrm{CDW}}$, again suggesting a limiting step in the reductive pathway.

\section{Conclusions}

The metabolic flux analysis of high-producing succinic acid yeast revealed a high recycling of the acid product over the cell membrane (about $15 \mathrm{mmol} / \mathrm{g}_{\mathrm{CDW}} / \mathrm{h}$ ) as well as exchange between the cytosol and mitochondria. Extracellular, labeled succinic acid was imported and labeled carbon atoms were found back in intermediates of the TCA cycle. Thermodynamic considerations, especially with respect to the required oxidative direction of fumarase in the mitochondria and reductive direction in the cytosol, suggests that malate is mostly present in the cytosol. The high intracellular concentration leads to excretion of malate to the extracellular space but also transport of malate into the mitochondria. Because there is correlation between the cytosolic/mitochondrial malate ratio and the exchange flux, the flux estimation has a comparably high standard deviation. Nevertheless, the labeling measurements require an exchange of malate between the cytosol and mitochondria.

The kinetics of fumarase have been studied in vitro and inhibition by inorganic phosphate has been described [29]. Recent measurements in wild-type strains suggest a cytosolic phosphate concentration of $20 \mathrm{mM}$, which is the range of the $K_{I}$ for phosphate inhibition [29]. Unfortunately, no phosphate measurements were available for the strain used here.

For the current work, several assumptions were derived from previous observations or theoretical considerations, rather than direct measurements. While these assumptions influence the concentration ratios and thermodynamic interpretation, the flux and amount ratio estimated from the labeling measurements is not influenced by these assumptions. During the parameter optimization a coefficient representing the amount (units $\mu \mathrm{mol} / \mathrm{g}$, not the compartment specific concentration) ratio was used. Furthermore, the intracellular fluxes were normalized to whole cell amounts (unit $\mu \mathrm{mol} / \mathrm{g} / \mathrm{h}$, not compartment specific volumes).
Compartment specific measurements would facilitate and improve the identification of fluxes and ratios. Similar to previous developed sensor reactions for (cytosolic) concentration measurements [30-32], sensor reactions mirroring the labeling enrichment could be introduced and used to obtain mitochondrial or cytosolic labeling enrichments. Further, physiological measurements, especially regarding the intracellular volume distribution under the observed conditions could reduce the amount of assumptions and increase the accuracy for data interpretation like thermodynamics. The distribution of the acids is also influenced by the electrochemical potential and $\mathrm{pH}$ difference which have not been measured under the cultivation conditions used here.

On the other hand, the labeling approach can overcome the limitation of direct compartment specific measurements to a certain extend and revealed intracellular product recycling. The measurements clearly show that compartmentation can lead to a condition of bidirectional fluxes, including the catabolism of the desired product. The labeling approach can easily be applied to other production processes and used to identify putative product recycling activity especially for other products derived from the TCA cycle or lactic acid production. Performing the labeling experiment in a secondary reactor like the bioscope furthermore reduces the amount of labeled material required and allows for a series of experiments with different substrate(s) labeling(s). The dilution approach allows to reach quasi steady-state conditions while the labeling state is switched, allowing to identify fast intracellular fluxes.

\section{Additional file}

Additional file 1. Supplementary information.

\section{Authors' contributions}

SAW wrote the manuscript and performed the ${ }^{13} \mathrm{C}$ mathematical modeling, CBM performed the bioreactor cultivations and measurements, ZZ, WMVG and $M L A J$ revised the manuscript and further strengthened the interpretation of the data. All authors read and approved the final manuscript.

\section{Author details}

${ }^{1}$ Department of Biotechnology, Delft University of Technology, Van der Maasweg 9, 2629 HZ Delft, The Netherlands. ${ }^{2}$ Present Address: Applikon Biotechnology B.V., Heertjeslaan 2, 2629 JG Delft, The Netherlands. ${ }^{3}$ DSM Biotechnology Center, Alexander Fleminglaan 1, 2613 AX Delft, The Netherlands.

\section{Acknowledgements}

The authors highly appreciate the excellent analytical work of A. ten Pierick, C. Ras and R. M. Seifar. Further we like to thank E. Nikerel for his valuable contribution in the initial phase of the project.

\section{Competing interests}

Authors M. L. A. Jansen and Z. Zhao are employed by DSM Biotechnology Center and have commercial interests in the production of succinic acid. 


\section{Availability of data and materials}

All data is available in the supplement or on request to the corresponding author. The used strain is property of DSM Biotechnology Center (Delft, the Netherlands).

All relevant data is documented in the manuscript (and figures) and the Additional file 1.

\section{Funding}

The project was funded by Nederlandse Organisatie voor Wetenschappelijk Onderzoek (NWO) in the framework of a Horizon Valorization grant.

\section{Publisher's Note}

Springer Nature remains neutral with regard to jurisdictional claims in published maps and institutional affiliations.

Received: 16 January 2017 Accepted: 12 May 2017

Published online: 23 May 2017

\section{References}

1. Jansen ML, van Gulik WM. Towards large scale fermentative production of succinic acid. Curr Opin Biotechnol. 2014;30:190-7.

2. van Maris AJ, Geertman JM, Vermeulen A, Groothuizen MK, Winkler AA Piper MD, van Dijken JP, Pronk JT. Directed evolution of pyruvate decarboxylase-negative Saccharomyces cerevisiae, yielding a C2-independent, glucose-tolerant, and pyruvate-hyperproducing yeast. Appl Environ Microbiol. 2004;70:159-66.

3. van Maris AJ, Konings WN, van Dijken JP, Pronk JT. Microbial export of lactic and 3-hydroxypropanoic acid: implications for industrial fermentation processes. Metab Eng. 2004;6:245-55.

4. Choi S, Song CW, Shin JH, Lee SY. Biorefineries for the production of top building block chemicals and their derivatives. Metab Eng. 2015;28:223-39.

5. Cheng KK, Wang GY, Zeng J, Zhang JA. Improved succinate production by metabolic engineering. Biomed Res Int. 2013;2013:538790.

6. Ahn JH, Jang YS, Lee SY. Production of succinic acid by metabolically engineered microorganisms. Curr Opin Biotechnol. 2016;42:54-66.

7. Kuhnert P, Scholten E, Haefner S, Mayor D, Frey J. Basfia succiniciproducens gen. nov., sp. nov., a new member of the family Pasteurellaceae isolated from bovine rumen. Int J Syst Evol Microbiol. 2010;60:44-50.

8. Lee SJ, Lee DY, Kim TY, Kim BH, Lee J, Lee SY. Metabolic engineering of Escherichia coli for enhanced production of succinic acid, based on genome comparison and in silico gene knockout simulation. Appl Environ Microbiol. 2005;71:7880-7.

9. Wendisch VF, Bott M, Eikmanns BJ. Metabolic engineering of Escherichia coli and Corynebacterium glutamicum for biotechnological production of organic acids and amino acids. Curr Opin Microbiol. 2006;9:268-74

10. Jantama K, Haupt MJ, Svoronos SA, Zhang X, Moore JC, Shanmugam KT, Ingram LO. Combining metabolic engineering and metabolic evolution to develop nonrecombinant strains of Escherichia coli $\mathrm{C}$ that produce succinate and malate. Biotechnol Bioeng. 2008;99:1140-53.

11. Los M. Minimization and prevention of phage infections in bioprocesses. Methods Mol Biol (Clifton, NJ). 2012;834:305-15.

12. Biosuccinium ${ }^{\mathrm{TM}}$ technology [http://www.reverdia.com/technology/ technology-overview/]. Accessed 16 May 2017

13. Yuzbashev TV, Yuzbasheva EY, Sobolevskaya TI, Laptev IA, Vybornaya TV, Larina AS, Matsui K, Fukui K, Sineoky SP. Production of succinic acid at low $\mathrm{pH}$ by a recombinant strain of the aerobic yeast Yarrowia lipolytica. Biotechnol Bioeng. 2010;107:673-82

14. Bioamber. https://www.bio-amber.com. Accessed 16 May 2017.

15. Cok B, Tsiropoulos I, Roes AL, Patel MK. Succinic acid production derived from carbohydrates: an energy and greenhouse gas assessment of a platform chemical toward a bio-based economy. Biofuels Bioprod Biorefining. 2014;8:16-29.

16. Jansen MLA, Heijnen JJ, Verwaal R. Process for preparing dicarboxylic acids employing fungal cells. 2013. Patent WO/2013/004670.
17. Guldener U, Heck S, Fielder T, Beinhauer J, Hegemann JH. A new efficient gene disruption cassette for repeated use in budding yeast. Nucleic Acids Res. 1996;24:2519-24.

18. Mashego MR, van Gulik WM, Vinke JL, Visser D, Heijnen JJ. In vivo kinetics with rapid perturbation experiments in Saccharomyces cerevisiae using a second-generation BioScope. Metab Eng. 2006;8:370-83.

19. Van Dam JC, Eman MR, Frank J, Lange HC, Van Dedem GWK, Heijnen SJ. Analysis of glycolytic intermediates in Saccharomyces cerevisiae using anion exchange chromatography and electrospray ionization with tandem mass spectrometric detection. Anal Chim Acta. 2002;460:209-18.

20. Douma RD, de Jonge LP, Jonker CT, Seifar RM, Heijnen JJ, van Gulik WM Intracellular metabolite determination in the presence of extracellular abundance: application to the penicillin biosynthesis pathway in Penicillium chrysogenum. Biotechnol Bioeng. 2010;107:105-15.

21. Canelas AB, ten Pierick A, Ras C, Seifar RM, van Dam JC, van Gulik WM, Heijnen JJ. Quantitative evaluation of intracellular metabolite extraction techniques for yeast metabolomics. Anal Chem. 2009;81:7379-89.

22. Barton PI, Pantelides CC. gPROMS-A combined discrete/continuous modeling environment for chemical processing systems. Simul Ser. 1993:25:25.

23. Aboka FO, Yang $H$, de Jonge LP, Kerste $R$, van Winden WA, van Gulik WM, Hoogendijk R, Oudshoorn A, Heijnen JJ. Characterization of an experimental miniature bioreactor for cellular perturbation studies. Biotechnol Bioeng. 2006;95:1032-42.

24. Niedenführ S, Pierick AT, van Dam PT, Suarez-Mendez CA, Noh K, Wahl SA. Natural isotope correction of MS/MS measurements for metabolomics and C fluxomics. Biotechnol Bioeng. 2015;113:1137-47. doi:10.1002/ bit.25859.

25. Canelas AB, Ras C, ten Pierick A, van Gulik WM, Heijnen JJ. An in vivo data-driven framework for classification and quantification of enzyme kinetics and determination of apparent thermodynamic data. Metab Eng. 2011;13:294-306

26. Jamalzadeh E. Taransport of dicarboxylic acids in Saccharomyces cerevisiae. Delft: Ph. D. Delft University of Technology; 2013.

27. Agrimi G, Russo A, Ricci MA. Role of mitochondrial carriers in metabolic engineering. J Bioprocess Biotech. 2014;4:164.

28. Mainguet SE, Gronenberg LS, Wong SS, Liao JC. A reverse glyoxylate shunt to build a non-native route from C4 to C2 in Escherichia coli. Metab Eng. 2013;19:116-27.

29. Mescam M, Vinnakota KC, Beard DA. Identification of the catalytic mechanism and estimation of kinetic parameters for fumarase. J Biol Chem. 2011:286:21100-9.

30. Zhang J, ten Pierick A, van Rossum HM, Seifar RM, Ras C, Daran JM, Heijnen JJ, Wahl SA. Determination of the cytosolic NADPH/NADP Ratio in Saccharomyces cerevisiae using shikimate dehydrogenase as sensor reaction. Sci Rep. 2015:5:12846.

31. Zhang J, Sassen T, Ten Pierick A, Ras C, Heijnen JJ, Wahl SA. A fast sensor for in vivo quantification of cytosolic phosphate in Saccharomyces cerevisiae. Biotechnol Bioeng. 2015;112:1033-46.

32. Canelas AB, van Gulik WM, Heijnen JJ. Determination of the cytosolic free $\mathrm{NAD} / \mathrm{NADH}$ ratio in Saccharomyces cerevisiae under steady-state and highly dynamic conditions. Biotechnol Bioeng. 2008;100:734-43.

33. Mo ML, Palsson BO, Herrgard MJ. Connecting extracellular metabolomic measurements to intracellular flux states in yeast. BMC Syst Biol. 2009;3:37.

34. Kakhniashvili D, Mayor JA, Gremse DA, Xu Y, Kaplan RS. Identification of a novel gene encoding the yeast mitochondrial dicarboxylate transport protein via overexpression, purification, and characterization of its protein product. J Biol Chem. 1997:272:4516-21.

35. Kaplan RS, Mayor JA, Gremse DA, Wood DO. High level expression and characterization of the mitochondrial citrate transport protein from the yeast Saccharomyces cerevisiae. J Biol Chem. 1995:270:4108-14.

36. Palmieri L, Vozza A, Agrimi G, De Marco V, Runswick MJ, Palmieri F, Walker JE. Identification of the yeast mitochondrial transporter for oxaloacetate and sulfate. J Biol Chem. 1999;274:22184-90.

37. Albert $H$, Dale EC, Lee $E$, Ow DW Site-specific integration of DNA into wild-type and mutant lox sites placed in the plant genome. Plant J. 1995:7(4):649-59. 